\title{
LETTER
}

\section{Atypical antipsychotics and delirium in critical care}

\author{
Matt P Wise*, Madhankumar Vijayakumar and Steve Bowden \\ See related research by Wan et al., http://ccforum.com/content/15/3/R159
}

Wan and colleagues recently reported a case series of patients with refractory delirium successfully treated with the atypical antipsychotic drug quetiapine. This class of medication is used infrequently by critical care physicians at present and offers a potentially useful treatment for a group of patients who are clinically challenging to manage. However, larger studies are required to evaluate both efficacy and adverse events in a critically ill population.

Wan and colleagues reported a case series of 17 patients with refractory delirium treated with the atypical antipsychotic quetiapine [1]. A reduction in the concomitant use of other medications and resolution of delirium was observed. A contemporary survey of UK physicians found haloperidol was the treatment of choice for first-line (74\%) and second-line (34\%) management of hyperactive delirium [2]. Atypical antipsychotics were rarely used for first-line treatment of hyperactive delirium and were used by just over $10 \%$ of physicians as second-line treatment [2].

Treating patients with delirium is challenging and atypical antipsychotic drugs have fewer extrapyramidal side effects than haloperidol. Wan and colleagues observed increased somnolence and transient hypotension associated with quetiapine administration [1]. However, larger studies are required to adequately assess both efficacy and adverse effects in critically ill patients. Most adverse event data originate in patients with psychiatric disease and include agitation, tachycardia, hypotension (especially in the elderly), QT prolongation, tardive dyskinesia, malignant neuroleptic syndrome, extrapyramidal effects, hepatitis, hyperglycaemia, hyponatraemia, dyslipdaemia, thromboembolism, pneumonia, and increased mortality in elderly patients with dementia-associated psychosis. These side effects may be more significant in critically ill patients with limited physiological reserve. Moreover, as atypical antipsychotics are extensively metabolised by cytochrome P450 , the potential for drug interactions increases, leading to either toxic or subtherapeutic levels [3]. Paradoxical agitation may be observed during initiation and rapid dose escalation with quetiapine [4]. This phenomenon is well described with benzodiazepines [5], which are used frequently for the second-line management of hyperactive agitation [2], and requires further characterisation in the critically ill.

\section{Authors' response}

Cathrine A McKenzie, Ruth YY Wan and Nicholas A Barrett

We thank Dr Wise and colleagues for their insightful comments. We agree that our retrospective case series provides useful research-generating data. We look forward to participating in future randomised controlled studies of atypical antipsychotics in the critically ill.

With regards to their comments on side effects, only limited sedation and transient hypotension were observed in the reported case series. Paradoxical agitation was not observed in the 17 patients described, and indeed has not been noted in further restricted use of

*Correspondence: mattwise@doctors.org.uk

Department of Critical Care, University Hospital of Wales, Cardiff CF14 4XW, UK [AU Query: Confirm affiliation] quetiapine within the critically ill at Guy's and St Thomas' NHS Foundation Trust. Whether this is because of the low doses of quetiapine used, the inherent bias that exists in retrospective observational studies or the small number of patients studied is difficult to fully establish. A single case of QT prolongation was described in the series and this was in association with the potential interacting agent fluconazole [1]. As with all prescriptions for agents where there is a risk of an interaction that can result in prolongation of QT, careful prescription assessment by the multidisciplinary team and regular 12-lead electrocardiograms may help minimise risk of drug-drug interactions and potential patient harm [6,7]. 
Published: 16 November 2011

\section{References}

1. Wan RYY, Kasliwal M, McKenzie CA, Barrett NA: Quetiapine in refractory hyperactive and mixed intensive care delirium: a case series. Crit Care 2011, 15:R159.

2. Mac Sweeney R, Barber V, Page V, Ely EW, Perkins GD, Young JD, McAuley DF Intensive Care Foundation: A national survey of the management of delirium in UK intensive care units. QJM 2010, 103:243-251.

3. Conley RR, Kelly DL: Drug-drug interactions associated with secondgeneration antipsychotics: considerations for clinicians and patients. Psychopharmacol Bull 2007, 40:77-97.

4. Smith MA, McCoy R, Hamer-Maansson J, Brecher M: Rapid dose escalation with quetiapine: a pilot study. J Clin Psychopharmacol 2005, 25:331-335.
5. Saïas T, Gallarda T: Paradoxical aggressive reactions to benzodiazepine use: a review. Encephale 2008, 34:330-336.

6. Leape LL, Cullen DJ, Clapp MD, Burdick E, Demonaco HJ, Erickson JI, Bates DW: Pharmacist participation on physician rounds and adverse drug events in the intensive care unit. JAMA 1999, 282:267-270.

7. Novotny T, Florianova A, Ceskova E, Weislamplova M, Palensky V, Tomanova J, Sisakova M, Toman O, Spinar J: Monitoring of QT interval in patients treated with psychotropic drugs. Int J Cardio/ 2007, 117:329-332

doi:10.1186/cc10493

Cite this article as: Wise MP, et al:: Atypical antipsychotics and delirium in critical care. Critical Care 2011, 15:453. 